\title{
BMJ Open Concerns and desires of healthcare workers caring for patients with COVID-19 in April and July 2020 in Japan: a qualitative study of open-ended survey comments
}

\author{
Mami Kayama (1) , ${ }^{1}$ Yumi Aoki, ${ }^{1}$ Takahiro Matsuo (D) ,2 Daiki Kobayashi (D) , ${ }^{3}$ \\ Fumika Taki ${ }^{4}$
}

To cite: Kayama M, Aoki Y, Matsuo T, et al. Concerns and desires of healthcare workers caring for patients with COVID-19 in April and July 2020 in Japan: a qualitative study of open-ended survey comments. BMJ Open 2022;12:e051335. doi:10.1136/ bmjopen-2021-051335

- Prepublication history and additional supplemental material for this paper are available online. To view these files, please visit the journal online (http://dx.doi.org/10.1136/ bmjopen-2021-051335).

Received 16 March 2021 Accepted 29 December 2021

Check for updates

(c) Author(s) (or their employer(s)) 2022. Re-use permitted under CC BY-NC. No commercial re-use. See rights and permissions. Published by BMJ.

For numbered affiliations see end of article.

Correspondence to

Dr Mami Kayama;

mkayama@sıcn.ac.jp

\section{ABSTRACT}

Objectives The prolonged effects of the COVID-19 pandemic continue to have a serious impact on healthcare workers. We described and compared the experiences of healthcare workers in Japan during the first wave of the COVID-19 pandemic from March to May 2020, and during the lull from June to July 2020.

Design In this qualitative study, we used a web-based survey to obtain comments from healthcare workers about their experiences during the pandemic, and explored these using inductive content analysis.

Setting A tertiary emergency hospital in Tokyo, in April and July 2020.

Participants Participants were staff in the hospital, including physicians, nurses, pharmacists, radiological technicians and laboratory medical technicians. Many, but not all, had directly cared for patients with COVID-19.

Results In total, 102 participants in the first survey and 154 in the second survey provided open-ended comments. Three themes were extracted: concerns, requests and gratitude. There were four subthemes under concerns: the hospital infection control system, fear of spreading infection to others, uncertainty about when the pandemic would end and being treated as a source of infection. There were 53 requests in the first survey and 106 in the second survey. These requests were divided into seven subthemes: compensation, staffing, information, facilities, leave time, PCR tests and equitable treatment. The theme on gratitude had two subthemes: information and emotional support, and material support. The fears and desires of healthcare workers included two types of uncertainty-related concerns, and requests were very different across the two surveys.

Conclusions it is important to apply a balance of information to help staff adjust to their new work environment, as well as support to minimise the burden of infection and impact on their families.

\section{INTRODUCTION}

Healthcare workers caring for patients during a pandemic are required to work under stressful conditions. ${ }^{1}$ In Japan, the first
Strengths and limitations of this study

- This is the first study to qualitatively explore frontline healthcare workers' experiences in Japan during the COVID-19 pandemic.

- This study described and compared the experiences of healthcare workers during the first wave of the COVID-19 pandemic, from March to May 2020, and during the lull from June to July 2020.

- The study sample was limited to acute care and a single institution.

- This was a short-term study with 3 months between the two surveys, and long-term experiences should be explored in the future.

case of the new COVID-19 was recorded on 16 January $2020 .^{2}$ The country then experienced a rapid spread of the infection, mainly in urban areas, and a state of emergency was declared between 7 April and 25 May 2020. ${ }^{2}$ Healthcare workers had to work with limited resources, and were at considerable risk of infection.

There is a growing body of descriptive research focusing on the experiences of healthcare workers under these conditions. For example, one study found that primary care physicians were more concerned about being unable to provide medical care if they were infected than about becoming sick themselves. ${ }^{3}$ Joo and Liu examined nurses' experiences, and identified several barriers to COVID-19 care, including (a) limited information about COVID-19, (b) unpredictable challenges and difficult practice, (c) inadequate support, (d) family concerns and (e) emotional and psychological stress. ${ }^{4}$

Matsuo and colleagues reported on the mental health of healthcare workers in the early stages of the pandemic. ${ }^{56}$ They analysed 
quantitative data on the level of burnout and related factors, through a survey at a single medical institution in Japan. These studies have been cited in many other papers as providing valuable data about the early stage of the pandemic in Japan. In this paper, we aimed to qualitatively analyse the free-text statements in the responses to the questionnaire used by Matsuo and colleagues. We hope that this will provide valuable information about risk management in the early stages of an unfolding disaster, by comparing data from the period when the number of infected people increased in the early stage of the pandemic in Japan with a quieter period a few months later.

\section{METHODS}

\section{Participants and setting}

The participants were drawn from a purposive sample of healthcare workers in a tertiary emergency medical hospital, St. Luke's International Hospital in Tokyo, Japan. By 15 June 2020, the hospital had treated more than 220 confirmed and 350 suspected patients with COVID-19, or $3.2 \%$ of the 5587 confirmed patients in Tokyo.

The participants in the first survey were physicians, nurses, laboratory medical technicians, radiological technicians and pharmacists, all of whom worked in departments that had direct contact with patients with COVID-19, including emergency care, general internal medicine, respiratory medicine, infectious diseases, general wards and intensive care units (ICUs). The participants in the second survey were all staff in the hospital, including physicians, nurses, laboratory medical technicians, radiological technicians, pharmacists, clinical engineering technicians, physical therapists, registered dieticians, medical clerks and receptionists.

\section{Data collection}

Data were collected through two online cross-sectional surveys of healthcare workers (online supplemental file 1), the first from 6 April to 19 April 2020 and the second from 15 June to 6 July 2020 at a tertiary hospital in Tokyo, Japan, with some of the highest numbers of patients with COVID-19 in Japan (figure 1).

The first survey was conducted before the government's decision to provide benefits for healthcare workers responding to the new COVID-19 infection. The second survey was conducted after the benefits had been paid.

\section{Data analysis}

We used inductive content analysis for the analysis. This approach allowed us to analyse our data without any predetermined themes. ${ }^{7}$ The advantage of an inductive approach is that the results become data-driven. ${ }^{8}$ With no preconceived ideas, this approach sharpened our ability to discern both differences and similarities in the data. ${ }^{8}$ First, we read and re-read the comments to get a general meaning and sense of the data. We then divided the texts into units of meaning, interpreted them, compared them, and categorised them into tentative subthemes, using the qualitative research software NVIVO12Pro. We then arranged, edited and formulated the subthemes into descriptive themes. We analysed the differences between the two surveys as well as the details of the freetext content. Five researchers were involved in the analysis of the free text: a hospital occupational physician, two infectious diseases physicians and two nurse researchers specialising in psychiatric and mental health nursing and qualitative research methods. This process aimed to provide a detailed analysis of the situation among healthcare workers, to contribute to planning support for the future. The two survey periods were at different times,

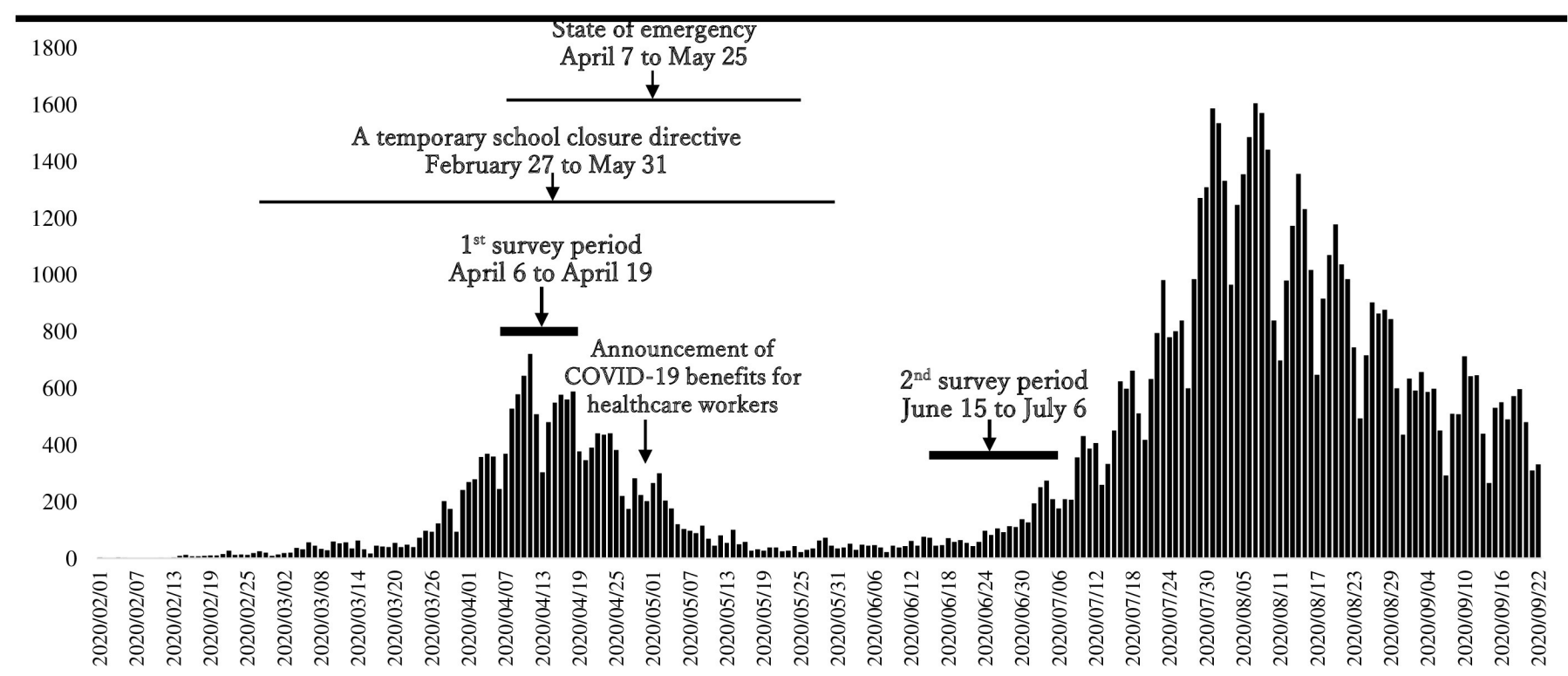

Figure 1 Events related to the study during the pandemic, mapped against number of daily confirmed COVID-19 cases in Japan. 
when the infection situation was different. We therefore thought that the data would enable us to examine the support required at different stages of the pandemic, with different levels of infection, and therefore varying pressure on healthcare workers. The researchers reviewed and discussed the results together to explore different perspectives.

\section{Data trustworthiness}

$\mathrm{Guba}^{9}$ used four criteria to evaluate the trustworthiness of data: credibility, dependability, confirmability and transferability. Anney also identified four criteria for ensuring credibility in qualitative research: prolonged engagement, triangulation, member checks, peer debriefing and negative case analysis. ${ }^{10}$ We used these criteria to ensure trustworthiness in our study. To ensure prolonged engagement, several of our research team were employed at the hospital. They therefore understood comments and concerns about the working environment, because they themselves have been dealing with concerns about infection control, and high levels of anxiety. To deliver triangulation, we drew on the quantitative data provided from the questionnaires to confirm our interpretations of the comments. Data analysis was shared among the researchers, and we were careful to obtain agreement about the interpretation of the free text.

\section{Ethical issues}

This survey was within a single facility, and we knew that it might be possible to identify individuals from their free-text comments, especially if they had included any information about their job title or work experience. To obtain honest opinions, the research team made clear that they would ensure that participants were not personally identifiable.

\section{Patient and public involvement}

No patients or members of the public were involved in the design or conduct of the study.

\section{RESULTS}

Of the 488 healthcare workers who were sent the first survey in April 2020, 369 (75.6\%) responded. Of these, 102 participants $(27.6 \%)$ wrote comments in response to the open-ended question. These were all included in the analysis. Among the 1672 healthcare workers, 672 (40.2\%) responded to the second survey, among whom 12 were excluded from the survey because of missing values. Of whom 660 responded, and 154 (23.3\%) included freetext comments, all of which were included the analysis. Of the 660 people responding to the second survey, 146 (22.1\%) had also responded to the first survey. Table 1 shows the demographic characteristics of the participants.

The content of the comments was divided into three themes: concerns, requests and gratitude. The following sections contain a description of each theme and subtheme, including similarities and differences observed across the two surveys. The number of data units extracted is shown in table 2.

\section{Concerns}

Hospital's infection control system

The largest number $(n=42)$ of statements were about the hospital's infection control system. In the first survey, many participants complained about the overwhelming lack of personal protective equipment (PPE). Responses described anxiety about the possibility that staff might not be adequately protected:

I'm worried that the lack of masks, shields, gloves, etc. used at work will increase the chance of infection. For example, using one mask for three days.

The second survey described their distress at the need for prolonged measures such as refraining from eating or talking together, and the investigations into their private lives if they were infected.

I feel there is a contradiction in the fact that restrictions on eating together and conversations with staff who work together on a regular basis are still in place, while the rest of the world has lifted restraints.

\section{Fear of spreading the infection to others}

In the first survey, fear of infection was mentioned by 12 respondents. Of these, 10 were concerned that they would be infected and 2 were concerned that they would spread the infection to others.

I see patients who deteriorate rapidly, and I don't want to take even the slightest chance that this will happen to me.

[I am worried about] risking the life of an elderly family member living with me if I take the virus home.

There was no mention of this concern in the second survey.

Uncertainty about the likely end of the infection

Uncertainty about the end of the infection was mentioned by 10 respondents in the first survey, but none in the second.

I have no idea when the situation will be resolved, and I am worried about what will happen if a nosocomial infection occurs.

\section{Being treated like a source of infection}

There were four complaints in the first survey about being treated like a source of infection by those around them. This issue was not raised in the second survey.

I'm embarrassed that I'm receiving so many COVID-19 patients that I'm sometimes treated like a source of infection by other departments and the general public. 
Table 1 Demographic characteristics

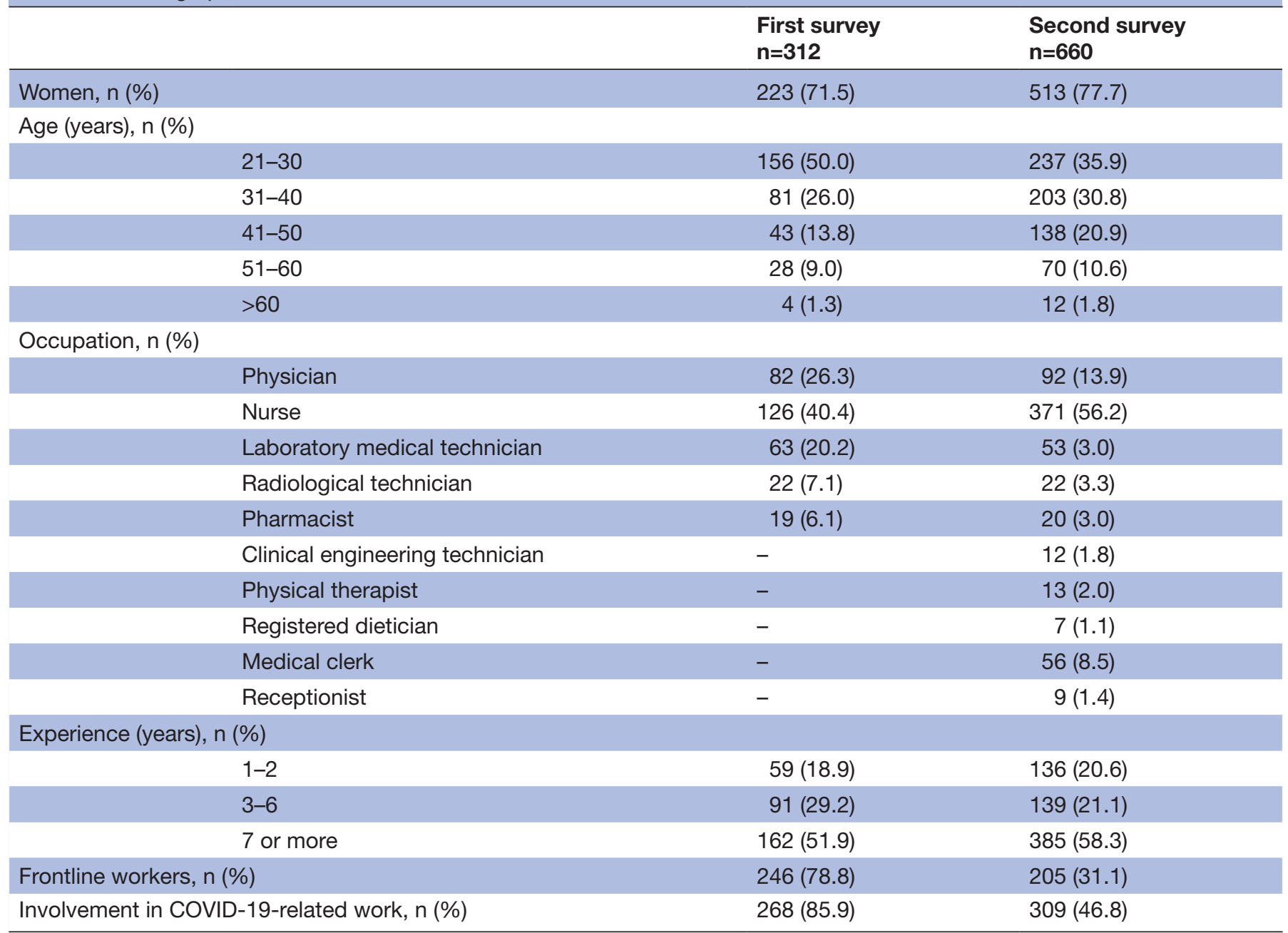

\section{Requests}

The respondents expressed many different requests, including 53 in the first survey, and 106 in the second survey.

\section{Compensation}

The most common request was for compensation $(n=52)$. Most of the respondents requested hazard pay for individuals or hospitals.

I think extra compensation for the general floor staff who are fighting on the frontline, especially ICU, respiratory, and infectious diseases, is a must.

Hospitals that accept patients should be subsidized.

The comments changed between the first and second surveys, reflecting the provision of special allowances (see figure 1).

There was no allowance for assistants who handled COVID, including supplies, cleaning, and installation, as well as the co-medical staff. I would like to see allowances for people other than doctors and nurses.

\section{Staffing}

The next most common request $(n=31)$ was for improved ward staffing. In the first survey, many respondents commented about the lack of staff available to care for patients with COVID-19 and the confusion about how to treat patients.

Insufficient staffing to care for critically ill patients.

By the second survey, specialised wards had been established, and ward functions reorganised within the hospital. Responses described the burden of caring for patients with different or unfamiliar diseases. There were also requests to improve the allocation of radiologists and the night shift system, and consideration for pregnant staff.

Only the COVID and medical wards were given human resource support, and the surgical wards were quite difficult [to manage].

\section{Information}

There were 20 requests for information. In the first survey, concerns were expressed about the lack of information 
Table 2 Number of descriptions of each theme

\begin{tabular}{|c|c|c|}
\hline Themes & First survey & Second survey \\
\hline Concerns & 67 & 10 \\
\hline The hospital's infection control system & 29 & 5 \\
\hline Uncertainty about the end of the infection & 11 & 0 \\
\hline Being treated like a source of infection & 4 & 0 \\
\hline Requests & 53 & 106 \\
\hline Compensation & 12 & 41 \\
\hline Staffing & 24 & 15 \\
\hline Information & 6 & 14 \\
\hline Facilities & 5 & 12 \\
\hline Equitable request & 2 & 6 \\
\hline Gratitude & 0 & 13 \\
\hline For information and emotional support & 0 & 2 \\
\hline For material support & 0 & 11 \\
\hline
\end{tabular}

about the infection situation in the hospital and the required administrative policies.

The status of infection in the hospital is not communicated at all. The hospital only sends out e-mails to enforce rules and requests, but does not communicate direction and guidelines at all.

In the second survey, some staff who had been transferred to new departments because of the reorganisation of ward functions expressed a desire to share information about COVID-19 infection control. Respondents also asked for information about the prospect of lifting the restrictions on hospital staff's freedom to eat out and travel, to match the situation in the wider community.

I would find it easier to understand the current situation in my office if the status of COVID patients or suspected patients in inpatient and outpatient settings were posted on the intranet on a daily basis.

\section{Facilities}

In the first survey, most of the requests were for a place for staff to stay if they did not wish to return home for fear of infecting their families.

Providing a place to stay to isolate them from the rest of the family.

In the second survey, the requests were for an environment for e-learning, checking the physical condition of visitors, establishing clear transport lanes for transporting patients and better control of temperature and humidity because it was hot when using PPE.
I need masks and gowns to control the temperature and humidity in the hot Ns [nurse's] station.

Secure a place to eat, work on medical records, and participate in meetings using Teams.

\section{Leave time}

Participants in wards that were COVID-19 compliant stated a desire for longer leave times to care for older family members and children. There was also mention of the need to provide special leave for staff who were deployed to support other departments or who were pregnant. Both of these requests occurred more frequently in the second survey.

Leave is needed even in wards where you are not directly involved; mental fatigue can be found in all areas and professions.

\section{PCR testing}

At the time of the first survey, staff who wanted to take a PCR test were not able to do so, and many expressed a desire for better access to testing.

We would like to have an environment where PCR tests are easily available.

The second survey included requests for clarification of procedures and testing standards, and also clarification of procedures and standards for inspections.

\section{Equitable treatment}

In the first survey, staff not directly caring for patients with COVID-19, but involved in logistical support, asked 
for acknowledgement and respect for their efforts. In the second survey, respondents talked about the inequity of the busier departments not being able to pick up various donated items such as lunch boxes. Respondents continued to request equitable treatment for departments providing logistical support.

\section{Gratitude}

In the first survey, respondents did not talk about feeling grateful. However, it was the first theme to emerge from the second survey. Respondents expressed gratitude for two main areas.

\section{For information and emotional support}

In the second survey, gratitude was expressed for the regular information provided by the infection control department, consultations being available 24/7, the extension of time for education of new staff, letters from volunteers and the administrative staff who maintained the outpatient waiting room.

We were very fortunate to have people from other departments who responded immediately to our ad hoc calls for support when we needed it.

\section{For material support}

In the second survey, staff expressed their gratitude for the support provided by donations, such as free vouchers for beauty salons and clothing. They also described their appreciation for the childcare support provided by the hospital and the support from other departments.

The support through donations was very much appreciated. It was very encouraging.

\section{DISCUSSION}

In this study, we aimed to describe and compare the experiences of healthcare workers during the first wave from March to May 2020 of the COVID-19 pandemic, and during the lull in hospital admissions from June to July 2020, at a tertiary hospital in Tokyo, Japan.

We found that the fears and desires of hospital staff included a sense of uncertainty. According to Mishel, uncertainty occurs in a situation in which it is impossible to assign a definite value to objects or events and/or predict outcomes accurately. ${ }^{11}$

There were two connected sources of uncertainty: the disease itself and the hospital work system. The descriptions of these two types of uncertainty-related concerns and requests differed greatly across the two surveys.

The first was about the infection itself and about how many people will be infected, and the risk to individual members of staff and their families and colleagues. Respondents' comments can basically be considered as an expression of the desire to regain control over the situation. Responding staff wanted the number of infected and suspected patients to be shown on the website every day. This is thought to be an expression of the desire to gain peace of mind by accurately grasping trends in the immediate environment. Previous researchers have also found that the lack of information and failure to properly update information has caused anxiety among healthcare workers during the COVID-19 pandemic. ${ }^{12}{ }^{13}$ There was little knowledge about the virus during the first wave, and therefore many healthcare workers showed fear of infection and uncertainty about the disease. There were greater shortages and reuse of PPE, especially N-95 masks, among ICU staff in Japan than internationally. ${ }^{14}$ In the quantitative analysis of the same survey, $31.4 \%$ (98 of 312) of staff were considered to be burned out in the first survey, with PPE deficiency having a statistically significant association. ${ }^{5}$

The second sense of uncertainty comes from the changes in the hospital environment required to treat infectious diseases. The first survey was at a time when the number of patients being admitted to medical institutions was increasing, and the functions of the wards were being reorganised to cope. This reorganisation was accompanied by staff reassignments and patient ward changes, which resulted in significant changes in staff care, procedures and relationships. Previous knowledge and procedures were often not applicable, making it harder for staff. Digby et al analysed responses to openended questions from healthcare workers in Melbourne Australia and also found themes of changing working conditions, working in the changed hospital environment, and personal isolation and uncertainty. ${ }^{15}$

Yamada et al found that one of the factors affecting the mental health of public health nurses after the Great East Japan Earthquake was the unclear nature of their work. ${ }^{16}$ The results of our study support knowledge about disaster and critical incident management gained from elsewhere in Japan during the pandemic and from the Great East Japan Earthquake. During both, the inability to spend time with peers and family members and the increased burden of daily life increased healthcare workers' psychological distress and fear of the disaster itself. ${ }^{17} 18$ Healthcare workers expressed anxiety about infecting family members, especially in the first survey. It has been reported in previous disasters that worries and events related to family members can be a major burden on the mental health of healthcare workers. ${ }^{19}$ Yonemoto highlighted the need to include mental healthcare measures for medical staff and their families in hospitals after the Great East Japan Earthquake. ${ }^{20}$ Umeda et al examined the roles of individuals and organisations before, during and after disasters in a review of studies and organisational reports from Japan and abroad. The roles of organisations during a disaster include providing appropriate leave, ensuring access to professional support and determining and responding to the possibility of continuing work in the field. ${ }^{21}$ Studies have also found that mental health education, work adjustments and workplace considerations are necessary to maintain the mental health of healthcare workers working under disaster conditions. ${ }^{20}{ }^{21}$ It is 
therefore important to apply a balance of information to help staff adjust to their new work environment, as well as support to minimise the burden of infection and impact on their families.

Gratitude was highlighted in the second survey. Mishel stated that when coping strategies are effective for an uncertain event appraised as either a danger or an opportunity, adaptation will occur. ${ }^{11}$ The expression of gratitude may indicate that people have successfully adapted to uncertainty. Sun et al suggested that nurses often showed early negative emotions in difficult circumstances, but gradually developed a feeling of gratitude to others, brought by respect from patients, support from colleagues and spending time with their families. ${ }^{22}$ When adequate support was available from colleagues, healthcare workers felt more appreciated and came to feel that they wanted to contribute more. ${ }^{23}$ Some studies also found that healthcare workers reported positive experiences during the COVID-19 pandemic, including good opportunities for personal growth and resilience, team unity, gaining experiential knowledge, and reflection on their lives. $^{2425}$

\section{Limitations}

This study had some limitations. First, it was conducted in a single institution among frontline healthcare workers in acute care settings, reducing its generalisability. Second, to minimise the further spread of COVID-19, we used an online survey with open-ended questions. This meant there were no opportunities for further probing, which would have been possible in face-to-face individual interviews. This was also a short-term study with only 3 months between the two surveys. Future studies should explore experiences in the longer term.

\section{CONCLUSIONS}

This study explored experiences during the first wave of COVID-19 in Japan, in spring 2020, and during the summer lull in the pandemic. Healthcare workers were concerned about the uncertainty of the situation, including fear of infection due to lack of PPE, worry about the impact on family members, and drastic changes in their work environment. To maintain the mental health of healthcare workers during disasters, prior mental health education, work adjustments and workplace considerations are necessary. It is important to apply a balance of information to help staff adjust to their new work environment, as well as support to minimise the burden of infection and impact on their families.

\footnotetext{
Author affiliations

${ }^{1}$ Department of Home Visit Nursing, St Luke's International Hospital,Graduate School of Nursing, St.Luke's International University, Chuo-ku, Japan ${ }^{2}$ Department of Infectious Diseases, St Luke's International Hospital, Chuo-ku, Japan

${ }^{3}$ Graduate School of Public Health, Division of General Internal Medicine, Department of Internal Medicine, St Luke's International University, Chuo-ku, Japan ${ }^{4}$ Department of Nephrology, St Luke's International Hospital, Chuo-ku, Japan
}

Acknowledgements We thank all participants of this survey.

Contributors All authors contributed towards the concept and design of the study. TM, DK and FT conducted the acquisition of the data through conducting the web-based surveys. MK conducted the qualitative analysis, with YA contributing the analysis. MK and YA prepared the first draft of the manuscript, with all authors contributing towards the review and approval of the final draft.

Funding This research received no specific grant from any funding agency in the public, commercial or not-for-profit sectors. The authors have not declared a specific grant for this research from any funding agency in the public, commercial or not-for-profit sectors.

Competing interests None declared.

Patient consent for publication Not required.

Ethics approval This study involves human participants and was approved by Institutional Review Board of St. Luke's International University in Tokyo, Japan (Number: 20-A078). Participants gave informed consent to participate in the study before taking part.

Provenance and peer review Not commissioned; externally peer reviewed.

Data availability statement All data relevant to the study are included in the article or uploaded as supplementary information. All data relevant to the study are included in the article.

Supplemental material This content has been supplied by the author(s). It has not been vetted by BMJ Publishing Group Limited (BMJ) and may not have been peer-reviewed. Any opinions or recommendations discussed are solely those of the author(s) and are not endorsed by BMJ. BMJ disclaims all liability and responsibility arising from any reliance placed on the content. Where the content includes any translated material, BMJ does not warrant the accuracy and reliability of the translations (including but not limited to local regulations, clinical guidelines, terminology, drug names and drug dosages), and is not responsible for any error and/or omissions arising from translation and adaptation or otherwise.

Open access This is an open access article distributed in accordance with the Creative Commons Attribution Non Commercial (CC BY-NC 4.0) license, which permits others to distribute, remix, adapt, build upon this work non-commercially, and license their derivative works on different terms, provided the original work is properly cited, appropriate credit is given, any changes made indicated, and the use is non-commercial. See: http://creativecommons.org/licenses/by-nc/4.0/.

\section{ORCID iDs}

Mami Kayama http://orcid.org/0000-0001-8945-8285

Takahiro Matsuo http://orcid.org/0000-0001-9389-0628

Daiki Kobayashi http://orcid.org/0000-0002-3641-2088

\section{REFERENCES}

1 Reger MA, Piccirillo ML, Buchman-Schmitt JM. COVID-19, mental health, and suicide risk among health care workers: looking beyond the crisis. J Clin Psychiatry 2020;81:20com13381.

2 Japanese Ministry of Health, Labour and Welfare. Open data. Available: https://www.mhlw.go.jp/stf/covid-19/open-data.html

3 Verhoeven V, Tsakitzidis G, Philips H, et al. Impact of the COVID-19 pandemic on the core functions of primary care: will the cure be worse than the disease? A qualitative interview study in Flemish GPs. BMJ Open 2020;10:e039674.

4 Joo JY, Liu MF. Nurses' barriers to caring for patients with COVID-19: a qualitative systematic review. Int Nurs Rev 2021;42:760-71.

5 Matsuo T, Kobayashi D, Taki F, et al. Prevalence of health care worker burnout during the coronavirus disease 2019 (COVID-19) pandemic in Japan. JAMA Netw Open 2020;3:e2017271.

6 Matsuo T, Taki F, Kobayashi D, et al. Health care worker burnout after the first wave of the coronavirus disease 2019 (COVID-19) pandemic in Japan. J Occup Health 2021;63:e12247.

7 Hsieh H-F, Shannon SE. Three approaches to qualitative content analysis. Qual Health Res 2005;15:1277-88.

8 Graneheim UH, Lindgren B-M, Lundman B. Methodological challenges in qualitative content analysis: a discussion paper. Nurse Educ Today 2017;56:29-34.

9 Guba EG. Criteria for assessing the trustworthiness of naturalistic inquiries. ECTJ 1981;29:7591.

10 Anney VN. Ensuring the quality of the findings of qualitative research: looking at trustworthiness criteria. J Emerg Trends Educ Res Policy Studies 2014;5:272-81.

11 Mishel MH. Uncertainty in illness. Image J Nurs Sch 1988;20:225-32. 
12 Fawaz M, Samaha A. The psychosocial effects of being quarantined following exposure to COVID-19: a qualitative study of Lebanese health care workers. Int J Soc Psychiatry 2020;66:560-5.

13 Sougou NM, Diouf JB, Diallo AA, et al. [Risk perception of COVID-19 pandemic among health care providers: qualitative study conducted at the King Baudoin Hospital in Guédiawaye, the first hospital faced with managing a community-acquired COVID-19 case in Senegal]. Pan Afr Med J 2020;37:23.

14 Unoki T, Tamoto M, Ouchi A, et al. Personal protective equipment use by healthcare workers in intensive care unit during the COVID-19 pandemic in Japan: comparative analysis with the PPE-SAFE survey. Acute Med Surg 2020;7:e584.

15 Digby R, Winton-Brown T, Finlayson F, et al. Hospital staff well-being during the first wave of COVID-19: staff perspectives. Int J Ment Health Nurs 2021;30:440-50.

16 Yamada $\mathrm{H}$, Yamaguchi $\mathrm{H}$, Yoshida $\mathrm{H}$. A survey report on mental and physical health and mental health measures of public health nurses dispatched to the areas affected by the great East Japan earthquake. Journal of Public Health Nurses 2015;71:140-7.

17 Nukui H, Midorikawa S, Murakami M, et al. Mental health of nurses after the Fukushima complex disaster: a narrative review. J Radiat Res 2018;59:ii108-13.

18 Asaoka H, Koido Y, Kawashima Y, et al. Post-Traumatic stress symptoms among medical rescue workers exposed to COVID-19 in Japan. Psychiatry Clin Neurosci 2020;74:503-5. (in Japanese).
19 Kobayashi M, Misawa T, Komagata Y. Stress response and related factors of nurses who participated in disaster relief activities. Journal of Japan Society of Disaster Nursing 2011;12:47-57.

20 Yonemoto K. Influence of post-earthquake experiences on attitudes toward leaving and changing jobs among doctors and nurses in a disaster-affected area: findings for disaster retention management in hospitals. Journal of the Japanese Society for Healthcare Management 2016;9:13-19.

21 Umeda M, Chiba R, Sasaki M. A literature review on psychosocial support for disaster responders: qualitative synthesis with recommended actions for protecting and promoting the mental health of responders. Int J Environ Res Public Health 2011;2020.

22 Sun N, Wei L, Shi S, et al. A qualitative study on the psychological experience of caregivers of COVID-19 patients. Am J Infect Control 2020;48:592-8.

23 Travers JL, Schroeder K, Norful AA, et al. The influence of empowered work environments on the psychological experiences of nursing assistants during COVID-19: a qualitative study. BMC Nurs 2020;19:98

24 Aughterson H, McKinlay AR, Fancourt D, et al. Psychosocial impact on frontline health and social care professionals in the UK during the COVID-19 pandemic: a qualitative interview study. BMJ Open 2021:11:e047353.

25 Eftekhar Ardebili M, Naserbakht M, Bernstein C, et al. Healthcare providers experience of working during the COVID-19 pandemic: a qualitative study. Am J Infect Control 2021;49:547-54. 